PROCEEDINGS OF THE

AMERICAN MATHEMATICAL SOCIETY

Volume 136, Number 7, July 2008, Pages 2575-2582

S 0002-9939(08)09209-5

Article electronically published on March 10, 2008

\title{
A SHARP ROGERS AND SHEPHARD INEQUALITY FOR THE $p$-DIFFERENCE BODY OF PLANAR CONVEX BODIES
}

\author{
CHIARA BIANCHINI AND ANDREA COLESANTI
}

(Communicated by Jon G. Wolfson)

\begin{abstract}
We prove a sharp Rogers and Shephard type inequality for the $p$ difference body of a convex body in the two-dimensional case, for every $p \geq 1$.
\end{abstract}

\section{INTRODUCTION}

A convex body is a non-empty convex compact subset of $\mathbb{R}^{n}$; let us indicate the set of convex bodies in $\mathbb{R}^{n}$ with $\mathscr{K}^{n}$. To each convex body $K$ we can associate in a biunique way its support function $h_{K}$ :

$$
h_{K}(u)=\sup \{\langle x, u\rangle \mid x \in K\}, \quad \text { for all } u \in \mathbb{R}^{n},
$$

where $\langle\cdot, \cdot\rangle$ is the standard scalar product. The support function is a fundamental tool since the main properties of the body can be deduced from it.

One of the most interesting aspects of convex geometry, i.e. the theory of convex bodies, are geometric inequalities. An important family of inequalities are those leading to estimate the volume of a special body associated with a convex body (for example the difference body or the reflection body) in terms of the volume of the body itself. A remarkable inequality of this type is the classical Rogers and Shephard inequality (see [15]) which asserts that for all $K \in \mathscr{K}^{n}$

$$
V_{n}(K+(-K)) \leq\left(\begin{array}{c}
2 n \\
n
\end{array}\right) V_{n}(K),
$$

and equality holds if and only if $K$ is a simplex. Here $V_{n}(K)$ denotes the $n$ dimensional volume of $K$ (i.e. the $n$-dimensional Lebesgue measure). The body $K+(-K)$ is called the difference body of $K$ and it is the Minkowski sum of $K$ and its reflected body with respect to the origin, $-K$. We recall more generally that the Minkowski sum of $K$ and $L \in \mathscr{K}^{n}$ is

$$
K+L=\left\{z \in \mathbb{R}^{n} \mid z=k+l, \quad k \in K, l \in L\right\} .
$$

Another inequality due to Rogers and Shephard ([16]) concerns the convex hull (here denoted by conv) of $K$ and $-K$, under the assumption that the origin $o$ belongs to $K$ :

$$
V_{n}(\operatorname{conv}(K \cup(-K))) \leq 2^{n} V_{n}(K),
$$

Received by the editors February 6, 2007.

2000 Mathematics Subject Classification. Primary 52A40; Secondary 52A10.

(C)2008 American Mathematical Society Reverts to public domain 28 years from publication 
where equality holds if and only if $K$ is a simplex with one vertex at the origin.

In [6] Firey introduced a new operation for convex bodies, called $p$-sum, which depends on the parameter $p \geq 1$ and extends the Minkowski sum. This notion originated the so-called Brunn-Minkowski-Firey theory, or $L_{p}$ Brunn-Minkowski theory of convex bodies. The $L_{p}$ Brunn-Minkowski theory can be seen as an interpolation of the classical Brunn-Minkowski theory ( $L_{1}$ theory) that arises from Minkowski sums of convex bodies and the study of convex hulls of sets $\left(L_{\infty}\right.$ theory). Although it does not have the same direct geometric interpretation as the $L_{1}$ and $L_{\infty}$ cases, the $L_{p}$ theory has been attracting a lot of attention from both geometers and analysts because it provides analytic tools for geometric problems and new connections between convex geometry and analysis. An account on the $L_{p}$ theory of convex bodies can be found in the papers [9, [10]; recent important developments of this theory are contained in [11, [12, 2], 3], 13, 7], 4].

Let us fix $K, L \in \mathscr{K}^{n}$ both containing the origin; the $p$-sum of $K$ and $L, K+{ }_{p} L$, is defined by its support function in the following way:

$$
h_{K{ }_{p} L}(u)=\left(h_{K}^{p}(u)+h_{L}^{p}(u)\right)^{\frac{1}{p}}, \quad u \in \mathbb{R}^{n} .
$$

This definition admits a natural extension to the case $p=\infty$ :

$$
h_{K+\infty} L(u)=\lim _{p \rightarrow \infty} h_{K+{ }_{p} L}(u)=\max \left\{h_{K}(u), h_{L}(u)\right\}, u \in \mathbb{R}^{n} .
$$

Note that the extremal values $p=1$ and $p=\infty$ correspond to the Minkowski sum and the convex hull of the union, respectively. Indeed one has

$$
h_{K+{ }_{1} L}(u)=h_{K}(u)+h_{L}(u)=h_{K+L}(u)
$$

and

$$
h_{K+\infty}(u)=\max \left\{h_{K}(u), h_{L}(u)\right\}=h_{\operatorname{conv}(K \cup L)} .
$$

As proved by Firey [6] the $p$-sum is monotone with respect to the parameter $p$ : for all $K, L \in \mathscr{K}^{n}$ such that $o \in K, L$, if $p \leq q$, then

$$
K+{ }_{q} L \subseteq K+{ }_{p} L .
$$

This implies that for all $p \geq 1$,

$$
\operatorname{conv}(K \cup L) \subseteq K+{ }_{p} L \subseteq K+L .
$$

Another simple inclusion is

$$
K+{ }_{p} L \subseteq 2^{\frac{1}{p}} \operatorname{conv}(K \cup L) .
$$

In particular, choosing $L=-K$ and using inequalities (1.1) and (1.2), we have

$$
V_{n}\left(K+{ }_{p}(-K)\right) \leq \min \left\{\left(\begin{array}{c}
2 n \\
n
\end{array}\right), 2^{n \frac{(1+p)}{p}}\right\} V_{n}(K) .
$$

A natural problem is then to find the best constant $c=c_{n, p}$, depending on $n$ and $p$, such that

$$
V_{n}\left(K+_{p}(-K)\right) \leq c_{n, p} V_{n}(K), \quad \text { for all } K \in \mathscr{K}^{n}, o \in K .
$$

In this paper we solve this problem in the planar case $n=2$, for every $p \geq 1$. 
Theorem 1.1. For every $p \geq 1$ there exists a constant $c_{p}$ such that

$$
V_{2}\left(K+{ }_{p}(-K)\right) \leq c_{p} V_{2}(K),
$$

for all $K \in \mathscr{K}^{2}$. In particular if $K$ is a triangle with one vertex at the origin, then equality holds.

An explicit expression of $c_{p}$ will be presented in Section 3 .

We will show the $p$-Rogers and Shephard inequality (1.4) as a consequence of a theorem about the $p$-sum of the so-called parallel chord movements of convex bodies.

A parallel chord movement is a special one-parameter family of convex bodies which can be seen as continuous deformations of a fixed convex body. More precisely, fix $K \in \mathscr{K}^{n}$ and a direction $v \in \mathbb{R}^{n}$, which is the direction of the movement. We move each chord of $K$ parallel to $v$ in that direction with a certain speed, and we consider the union of these chords as the time parameter varies. If the speed function is suitably chosen, namely if the union of the chords is convex for all values of the parameter, then the family of the resulting convex bodies is a parallel chord movement.

Parallel chord movements are special cases of a wider class of movements of convex bodies introduced by Rogers and Shephard in [17, which have recently been applied in the proof of several inequalities in convex geometry (see, for example, [1], 2]-[5], 14]).

The importance of these movements is due principally to the behaviour of several geometric functionals with respect to the parameter of the movement. Indeed many of them, and the volume is the main example, are convex functions of the time parameter of the movement.

In particular in this paper we prove that if $K_{t}$ is a parallel chord movement, then the volume of its $p$-difference body $V_{n}\left(K_{t}+{ }_{p}\left(-K_{t}\right)\right)$ is a convex function of $t$, for all $p \geq 1$. This result, together with a technique used in [1, leads to the proof of Theorem 1.1. As noted in [1 this technique is successful only in the planar case, so our method cannot be used to prove inequality (1.3) in the general case $n \geq 2$.

The paper is organized as follows. In Section 2 we introduce several kinds of movements of convex bodies and we show some of their properties. Next to basic results we present a theorem about the $p$-sum of a particular type of movement. In section 3 we prove Theorem 1.1 as an application of the results concerning movements of convex bodies.

The authors wish to thank P. Gronchi for his suggestions and the referee for her or his helpful comments on this paper.

\section{Shadow Systems and linear Parameter Systems}

A shadow system is a family of $n$-dimensional convex bodies $\{K(u)\}$ obtained as the projection of a fixed convex body $\tilde{K} \subseteq \mathbb{R}^{n+1}$ onto the hyperplane $\left\{e_{n+1}^{\perp}\right\}$, which we identify with $\mathbb{R}^{n}$, along the direction $e_{n+1}+u$. Here $u$ varies in $\left\{e_{n+1}^{\perp}\right\}$. The shadow system is said to be originated from the $(n+1)$-dimensional body $\tilde{K}$.

A linear parameter system is a family of convex bodies $\left\{K_{t}\right\}$ that can be written in the form

$$
K_{t}=\operatorname{conv}\left\{x_{i}+\lambda_{i} t v: i \in I\right\}, \quad t \in \mathscr{I},
$$


where $I$ is an arbitrary index set, $\left\{x_{i}\right\}_{i \in I}$ and $\left\{\lambda_{i}\right\}_{i \in I}$ are bounded subsets of $\mathbb{R}^{n}$ and of $\mathbb{R}$ respectively, $\mathscr{I}$ is an interval of $\mathbb{R}$ and $v \in \mathbb{R}^{n}$ is the direction of the linear parameter system.

Linear parameter systems are shadow systems in which $u$ lies on a line; indeed we have the following result.

Proposition 2.1. $\left\{K_{t}\right\}_{t \in \mathscr{I}}$ is a linear parameter system in $\mathbb{R}^{n}$ if and only if there exist a convex body $\tilde{K}$ in $\mathbb{R}^{n+1}$ and $v \in\left\{e_{n+1}^{\perp}\right\}$ such that for every $t \in \mathscr{I}, K_{t}$ is the projection of $\tilde{K}$ onto the hyperplane $\left\{e_{n+1}^{\perp}\right\}$ along the direction $e_{n+1}-t v$.

The idea to view linear parameter systems as projections of higher-dimensional convex bodies is contained in the original papers by Rogers and Shephard ([17, [19]) and was largely used by Campi and Gronchi $([2]-[5])$. For the sake of completeness here we present the proof of Proposition 2.1.

Proof. Let $K_{t}$ be of the form (2.1) and let us define the body $\tilde{K}$ as follows:

$$
\tilde{K}=\operatorname{conv}\left(\left\{x_{i}+\lambda_{i} e_{n+1}: i \in I\right\}\right) .
$$

For all $t \in \mathscr{I}$ let us call $L_{t}$ the projection of $\tilde{K}$ onto $\left\{e_{n+1}^{\perp}\right\}$ along $e_{n+1}-t v$. For all $y \in L_{t}$ there exists $z \in \tilde{K}$ such that $y=z-\left\langle z, e_{n+1}\right\rangle\left(e_{n+1}-t v\right)$. Furthermore there exist $a_{i} \in e_{n+1}^{\perp}, \lambda_{i} \in \mathbb{R}$, and $\sigma_{i} \geq 0, i=1, \ldots, n+1$, such that $\sum_{i=1}^{n+1} \sigma_{i}=1$ and

$$
z=\sum_{i=1}^{n+1} \sigma_{i}\left(a_{i}+\lambda_{i} e_{n+1}\right)
$$

Therefore

$$
y=\sum_{i=1}^{n+1} \sigma_{i}\left(a_{i}+\lambda_{i} t v\right) .
$$

This implies that $L_{t}$ is contained in $K_{t}$. To prove the reverse inclusion one can observe that the previous implications are true in both directions.

Conversely, let $\tilde{K}$ be any $(n+1)$-dimensional convex body and fix $t \in \mathscr{I}$; its projection onto $\left\{e_{n+1}^{\perp}\right\}$ along $e_{n+1}-t v$ is the set

$$
L_{t}=\left\{e_{n+1}^{\perp}\right\} \cap\left\{x \in \mathbb{R}^{n+1} \mid x=z+s\left(e_{n+1}-t v\right), z \in \tilde{K}, s \in \mathbb{R}\right\} .
$$

This is equivalent to

$$
L_{t}=\left\{z-\left\langle z, e_{n+1}\right\rangle e_{n+1}+t\left\langle-z, e_{n+1}\right\rangle v: z \in \tilde{K}\right\}
$$

and, by the convexity of $\tilde{K},\left\{L_{t}\right\}_{t \in \mathscr{I}}$ is a linear parameter system as defined in (2.1).

From the previous proof it follows that the body $\tilde{K}$ which generates a linear parameter system of the form (2.1) can be explicitly written as

$$
\tilde{K}=\operatorname{conv}\left\{x_{i}+\lambda_{i} e_{n+1}: i \in I\right\} .
$$

Campi and Gronchi showed in [4] the following formula, which relates the support functions of $K_{t}$ and $\tilde{K}$ :

$$
h_{K_{t}}(u)=h_{\tilde{K}}\left(u+t\langle u, v\rangle e_{n+1}\right), \quad u \in \mathbb{R}^{n}, t \in \mathscr{I} .
$$


We can give a cinematic interpretation of a linear parameter system viewing the numbers $\lambda_{i}$ as the speeds of the points $x_{i}$ along the direction $v$ and $t$ as the time parameter.

If the index set $I$ is a convex body $K \in \mathscr{K}^{n}$ and the speed is a function of the point, then the linear parameter system is called continuous movement:

$$
K_{t}=\operatorname{conv}\{x+\alpha(x) t v: x \in K\}, \quad t \in \mathscr{I},
$$

where $\alpha(\cdot)$ is a bounded function on $K$.

Assume that the speed function is constant on each chord parallel to $v$, i.e. $\alpha(x)=\beta\left(x \mid v^{\perp}\right)$, where $x \mid v^{\perp}$ is the projection of $x$ onto $\left\{v^{\perp}\right\}$ and $\beta$ is a function defined on the ortoghonal projection of $K$ onto $\left\{v^{\perp}\right\}$. Moreover, if $\beta$ is such that convexity is preserved for any $t$, namely

$$
\left\{x+\beta\left(x \mid v^{\perp}\right) t v: x \in K\right\}=\operatorname{conv}\left\{x+\beta\left(x \mid v^{\perp}\right) t v: x \in K\right\},
$$

then the continuous movement is called parallel chord movement.

In other words a parallel chord movement is obtained by assigning to each chord parallel to the direction $v$ a speed vector $\beta\left(x \mid v^{\perp}\right) v$ and considering for each fixed time $t$ the union of these chords; such a union has to be convex. If for some value of $t \in \mathscr{I}$ it is not convex, then its convex hull is just a continuous movement but not a parallel chord movement (usually in this case one considers only the subset $\mathscr{J}$ of $\mathscr{I}$ such that $K_{t}$ is convex for $t \in \mathscr{J}$ ). The reason why we consider only convex unions of chords is due to the behaviour of the volume. Indeed if $\left\{K_{t}\right\}_{t \in \mathscr{I}}$ is a parallel chord movement, then the volume of $K_{t}$ is independent of $t$.

The following theorem is due to Rogers and Shephard (see [17]), and it is one of the main motivations for the use of linear parameter systems in the theory of convex bodies.

Theorem 2.2. The volume $V_{n}\left(K_{t}\right)$ of a linear parameter system is a convex function of the parameter $t$.

In 4 it is proved that the Minkowski sum of linear parameter systems is a linear parameter system. Here we extend this result to the $p$-sum. This fact is one of the main ingredients in the proof of the $p$-Rogers and Shephard inequality.

Theorem 2.3. Let $\left\{K_{t}\right\}_{t \in \mathscr{I}}$ and $\left\{L_{t}\right\}_{t \in \mathscr{I}}$ be linear parameter systems along the direction $v$ and let $p \geq 1$. Then $\left\{K_{t}+_{p} L_{t}\right\}_{t \in \mathscr{I}}$ is also a linear parameter system along the direction $v$.

The proof is a straightforward consequence of Proposition 2.1 and the following lemma.

Lemma 2.4. Let $\left\{K_{t}\right\}_{t \in \mathscr{I}}$ and $\left\{L_{t}\right\}_{t \in \mathscr{I}}$ be linear parameter systems along the same direction $v$ and let $\tilde{K}$ and $\tilde{L}$ be the $(n+1)$-dimensional convex bodies which generate $K_{t}$ and $L_{t}$, respectively, defined as in (2.2). Hence for all $t \in \mathscr{I}, K_{t}+{ }_{p} L_{t}$ is the projection of $\tilde{K}+{ }_{p} \tilde{L}$ onto the hyperplane $\left\{e_{n+1}^{\perp}\right\}$ along the direction $e_{n+1}-t v$.

Proof. Using (2.3) one has

$$
\begin{aligned}
h_{\tilde{K}{ }_{p} \tilde{L}}^{p}\left(u+t\langle u, v\rangle e_{n+1}\right) & =h_{\tilde{K}}^{p}\left(u+t\langle u, v\rangle e_{n+1}\right)+h_{\tilde{L}}^{p}\left(u+t\langle u, v\rangle e_{n+1}\right) \\
& =h_{K_{t}}^{p}(u)+h_{L_{t}}^{p}(u)=h_{K_{t}+{ }_{p} L_{t}}^{p}(u) .
\end{aligned}
$$


This implies that $K_{t}+{ }_{p} L_{t}$ is the projection of the body $\tilde{K}+{ }_{p} \tilde{L}$ onto the hyperplane $\left\{e_{n+1}^{\perp}\right\}$ along the direction $e_{n+1}-t v$, which means, by Proposition 2.1, that $K_{t}+{ }_{p} L_{t}$ is a linear parameter system along $v$.

\section{The Proof of the $p$-Rogers AND Shephard InEQUality}

Let us call $\mathscr{K}_{0}^{n}$ the set of convex bodies with non-empty interior and containing the origin, and let us consider the functional $F_{p}$ defined on $\mathscr{K}_{0}^{n}$ :

$$
F_{p}(K)=\frac{V_{n}\left(K+_{p}(-K)\right)}{V_{n}(K)} .
$$

It is clear that the best constant $c_{n, p}$ such that (1.3) holds is the supremum of $F_{p}$ in $\mathscr{K}_{0}^{n}$.

We will use linear parameter systems to find a maximum for the functional $F_{p}$ in the planar case. The starting point is the next proposition which follows from Theorem 2.2 and Theorem 2.3 and the fact that the volume is constant on each parallel chord movement.

Proposition 3.1. If $K_{t}$ is any parallel chord movement such that $K_{t} \in \mathscr{K}_{0}^{n}$ for all $t \in \mathscr{I}$, then $F_{p}\left(K_{t}\right)$ is a convex function of the parameter $t$.

In [1] the following fact is proved: if $P$ is a planar convex polygon with $m$ vertices, $m>3$, then there exists a parallel chord movement $\left\{P_{t}\right\}_{t \in\left[t_{0}, t_{1}\right]}$, with $t_{0}<0<t_{1}$, such that $P=P_{0}$ and $P_{t_{0}}$ and $P_{t_{1}}$ have at most $(m-1)$ vertices. By Proposition 3.1 it follows that

$$
F_{p}(P) \leq \max \left\{F_{p}\left(P_{t_{0}}\right), F_{p}\left(P_{t_{1}}\right)\right\} .
$$

Using this fact recursively we deduce that

$$
\sup _{\mathscr{P}} F_{p}=\sup _{\mathscr{T}} F_{p}
$$

where $\mathscr{P}=\left\{K \in \mathscr{K}_{0}^{2} \mid K\right.$ is a polygon $\}$ and $\mathscr{T}=\left\{K \in \mathscr{K}_{0}^{2} \mid K\right.$ is a triangle $\}$. Moreover, by the continuity of $F_{p}(\cdot)$ and a standard density argument, one has

$$
\sup _{\mathscr{K}_{0}^{2}} F_{p}=\sup _{\mathscr{T}} F_{p}
$$

In particular we are going to show that triangles with one vertex at the origin are maximizers for $F_{p}$. In order to do this, let $T \in \mathscr{T}$ and assume $o \in \operatorname{int}(T)$ (int denotes the interior). Then there exists a parallel chord movement (whose elements are translates of $T$ ), $\left\{T_{t}\right\}_{t \in\left[t_{0}, t_{1}\right]}$ with $t_{0}<0<t_{1}$, such that $T_{0}=T$ and $o \in \operatorname{bd}\left(T_{t_{0}}\right), o \in \operatorname{bd}\left(T_{t_{1}}\right)$ (bd denotes the boundary). Similarly, if $o \in \operatorname{bd}(T)$, then there exists a parallel chord movement containing $T$, whose endpoints are triangles with one vertex at $o$. Again using Proposition 3.1, we have proved that

$$
\sup _{\mathscr{K}_{0}^{2}} F_{p}=\sup _{\mathscr{T}_{0}} F_{p}
$$

where $\mathscr{T}_{0}$ is the set of triangles with one vertex at the origin.

Note that $F_{p}$ is invariant under non-singular linear transformations. This implies that $F_{p}$ is constant on $\mathscr{T}_{0}$.

This argument proves the following result.

Theorem 3.2. If $T$ is a triangle in $\mathscr{K}_{0}^{2}$ with one vertex at the origin, then $T$ is a maximizer for $F_{p}$. 
To compute the best constant $c_{2, p}$, we can choose as a maximizer the triangle with vertices at the origin, at $(1,0)$ and $(0,1)$; let us indicate it with $\bar{K}$. Namely

$$
c_{2, p}=\frac{V_{2}\left(\bar{K}+{ }_{p}(-\bar{K})\right)}{V_{2}(\bar{K})} .
$$

Then to express the value of $c_{2, p}$ it is necessary to know how the $p$-difference body $\bar{K}+_{p}(-\bar{K})$ looks. Here we use the parametrization of the boundary of a convex body in terms of its support function (see [18, Corollary 1.7.3]).

The support function of $\bar{K}+_{p}(-\bar{K})$ is

$$
h_{\bar{K}+_{p}(-\bar{K})}(w)= \begin{cases}\cos \theta & \text { if } 0 \leq \theta<\frac{\pi}{4}, \\ \sin \theta & \text { if } \frac{\pi}{4} \leq \theta<\frac{\pi}{2}, \\ \left(\sin ^{p} \theta+(-\cos \theta)^{p}\right)^{\frac{1}{p}} & \text { if } \frac{\pi}{2} \leq \theta \leq \pi,\end{cases}
$$

where $w=\mathbf{e}^{i \theta} \in S^{1}$. Furthemore, by the symmetry of $\bar{K}+_{p}(-\bar{K})$,

$$
h_{\bar{K}+_{p}(-\bar{K})}\left(\mathbf{e}^{i \theta}\right)=h_{\bar{K}+_{p}(-\bar{K})}\left(\mathbf{e}^{i(\theta-\pi)}\right),
$$

for all $\pi \leq \theta \leq 2 \pi$. Then a parametrization for the boundary of $\bar{K}+_{p}(-\bar{K})$, for $1<p<+\infty$, is $\zeta(\theta)=(x(\theta), y(\theta))$, where

$$
\begin{gathered}
x(\theta)=\left\{\begin{array}{lc}
1-\frac{2}{\pi} \theta & \text { for } \theta \in\left[0, \frac{\pi}{2}\right], \\
-\left(\sin ^{p} \theta+(-\cos \theta)^{p}\right)^{\frac{1-p}{p}}(-\cos \theta)^{p-1} & \text { for } \theta \in\left(\frac{\pi}{2}, \pi\right),
\end{array}\right. \\
y(\theta)= \begin{cases}\frac{2}{\pi} \theta & \text { for } \theta \in\left[0, \frac{\pi}{2}\right], \\
\left(\sin ^{p} \theta+(-\cos \theta)^{p}\right)^{\frac{1-p}{p}} \sin ^{p-1} \theta & \text { for } \theta \in\left(\frac{\pi}{2}, \pi\right),\end{cases}
\end{gathered}
$$

and the remaining part of the boundary can be found using the symmetry of the body.

A picture can perhaps better show the geometry of the body. In Figure 1, $\bar{K}+_{p}(-\bar{K})$ is represented for the the values $1,1.5,2,15, \infty$ of the parameter $p$.

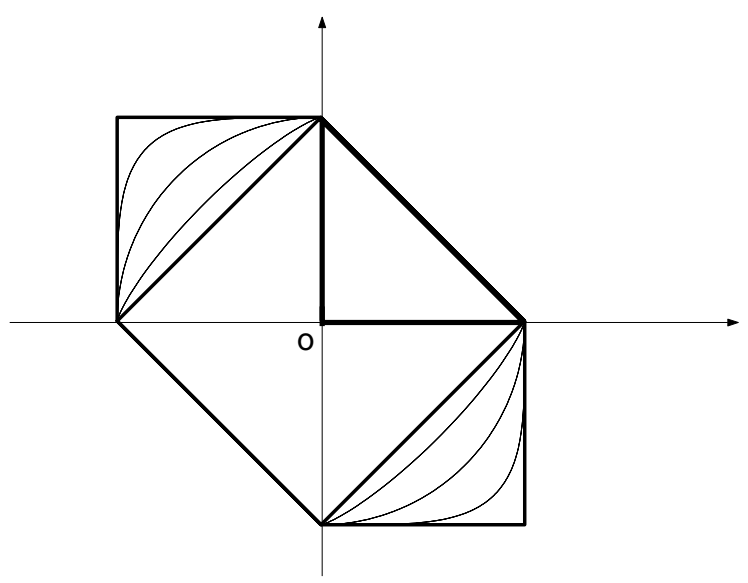

FIGURE 1 
Using the above parametrization and Gauss-Green's formulas we can express the area of $\bar{K}+{ }_{p}(-\bar{K})$ and then the value of the best constant $c_{2, p}$ :

$$
c_{2, p}=2\left(1+(p-1) \int_{0}^{\frac{\pi}{2}} \frac{\sin ^{p-2} t \cos ^{p-2} t}{\left(\sin ^{p} t+\cos ^{p} t\right)^{2 \frac{(p-1)}{p}}} d t\right), \quad 1<p<+\infty .
$$

\section{REFERENCES}

[1] S. Campi, A. Colesanti, P. Gronchi, A note on Sylvester's problem for random polytopes in a convex body, Rend. Ist. Mat. Univ. Trieste 31 (1999), 79-94. MR1763244(2001d:52014)

[2] S. Campi, P. Gronchi, The $L^{p}$-Busemann-Petty centroid inequality, Adv. Math. 167 (2002), 128-141. MR1901248(2003e:52011)

[3] - On the reverse $L^{p}$-Busemann-Petty centroid inequality, Mathematika 49 (2002), 1-11. MR2059037(2005d:52006)

[4] - On volume product inequalities for convex sets, Proc. Amer. Math. Soc. 134, 8 (2006), 2393-2402. MR2213713 (2007a:52010)

[5] Volume inequalities for $L_{p}$-zonotopes, Mathematika 53 (2006), 71-80. MR 2304053 (2007m:52011)

[6] WM. J. Firey, p-Means of convex bodies, Math. Scand. 10 (1962), 17-24. MR0141003 $(25: 4416)$

[7] C. Hu, X.-N. Ma, C. Schen, On the Christoffel-Minkowski problem of Firey's p-sum, Calc. Var. Partial Diff. Eqns. 21 (2004), 137-155. MR2085300 (2005g:52019)

[8] F. John, Extremum problems with inequalities as subsidiary conditions, Courant Anniversary Volume, Interscience, New York, 1948, 187-204. MR0030135(10:719b)

[9] E. Lutwak, The Brunn-Minkowski-Firey theory. I. Mixed volumes and the Minkowski problem, J. Differential Geom. 38 (1993), 131-150. MR.1231704 (94g:52008)

[10] _ The Brunn-Minkowski-Firey theory. II. Affine and geominimal surface areas, Adv. Math. 118, 2 (1996), 244-294. MR.1378681 (97f:52014)

[11] E. Lutwak, D. Yang, G. Zhang, $L_{p}$ affine isoperimetric inequalities, J. Differential Geom. 56 (2000), 111-132. MR.1863023 (2002h:52011)

[12] Sharp affine $L_{p}$ Sobolev inequalities, J. Differential Geom. 62 (2002), 17-38. MR.1987375 (2004d:46039)

[13] , On the $L_{p}$-Minkowski problem, Trans. Amer. Math. Soc. 356 (2004), 4359-4370. MR2067123 (2005d:52013)

[14] M. Meyer, S. Reisner, Shadow systems and volumes of polar convex bodies, Mathematika 53 (2006), 129-148. MR 2304056 (2008d:52010)

[15] C.A. Rogers, G.C. ShePhard, The difference body of a convex body, Arch. Math. 8 (1957), 220-233. MR0092172(19:1073f)

[16] Convex bodies associated with a given convex body, J. Lond. Math. Soc. 33 (1958), 270-281. MR0101508 (21:318)

[17] _ Some extremal problems for convex bodies, Mathematika 5 (1958), 93-102. MR0104203 (21:2960)

[18] R. Schneider, Convex bodies: the Brunn-Minkowski theory, Encyclopedia of Mathematics and Its Applications 44, Cambridge University Press, Cambridge, 1993. MR 1216521 (94d:52007)

[19] G.C. ShePhArd, Shadow system of convex sets, Israel J. Math. 2 (1964), 229-236. MR0179686 $(31: 3931)$

Dipartimento di Matematica 'U. Dini', Università degli Studi di Firenze, Viale MorGAGNi 67/A, 50134 Firenze, ItAly

E-mail address: chiara.bianchini@math.unifi.it

Dipartimento di Matematica 'U. Dini', Università degli Studi di Firenze, Viale MorGAGNi 67/A, 50134 Firenze, ITALy

E-mail address: colesant@math.unifi.it 